\title{
Numerical solution of singular Fredholm integral equations of the first kind using Newton interpolation
}

\author{
E.S. Shoukralla*, W. A. Elganaini**, and M. A. Markos*** \\ * Dept. of Physics and Engineering Mathematics, Faculty of Engineering and \\ Technology, Future University. \\ **, *** Dept. of Physics and Engineering Mathematics, Faculty of Electronic \\ Engineering, Menoufia University.
}

(Received: 20 May 2018 - Accepted: 27 May 2018)

\begin{abstract}
In this paper a computational technique is presented for the numerical solution of a certain potential-type singular Fredholm integral equation of the first kind with singular unknown density function, and a weakly singular logarithmic kernel. This equation is equivalent to the solution of the Dirichlet boundary value problem for Laplace equation for an open contour in the plane. The parameterization of the open contour facilitates the treatment of the density function's singularity in the neighborhood of the end-points of the contour, and the kernel's singularity. The unknown density function is replaced by a product of two functions; the first explicitly expresses the bad behavior of the density function, while the second is a regular unknown function, which will be interpolated using Newton interpolation in a matrix form. The singularity of the parameterized kernel is treated by expanding the two argument parametric functions into Taylor polynomial of the first degree about the singular parameter. Moreover, two asymptote formulas are used for the approximation of the kernel. In addition, an adaptive Gauss-Legendre formula, is applied for the computations of the obtained convergent integrals. Thus the required numerical solution is found to be equivalent to the solution of a system of algebraic equations. The numerical solution of the illustrated example is closer to the exact solution; which ensures the high accuracy of the presented computational technique.
\end{abstract}

Keywords: Electro-optics, electromagnetism, Fredholm integral equations, well-posed, singular, logarithmic kernel. 


\section{Introduction}

This paper is devoted to establish a computational technique for the numerical solution of a certain potential-type Fredholm integral equation of the first kind, whose unknown function (density) is singular in the neighborhood of the integration domain, and has a weakly singular kernel. In section 2, the reformulating of the Dirichlet problem for the Laplace equation of a mass distribution on an open contour in the plane is given. The equivalent potential-type Fredholm integral equation of the first kind is derived. In particular, Eq. (2) of section 2, arises in the fields of electron-optics, potential theory, and electromagnetism $[1,2]$ in the case of the replacement of a harmonic function by single-layer potentials. The finite energy condition, and the condition that the potential function tends to a constant at infinity are also imposed for the stability of the solution of the Dirichlet problem.

It should be noted that many methods [3-11] have been published for the solution of such equation, while the goal of the presented paper is to give an innovative approach for the numerical solution of this potential-type in the case of an open contour in the plane, in such a manner that the singularities of the density function disappear upon interpolating it via Newton Interpolation in matrix forms.

Actually, the approximation of the density function is based on replacing it by a product of two functions, the first is "badly behaved"; since it explicitly represents the singularity of the original singular density functions, while the second is a regular unknown function. The regular unknown function is approximated by Newton interpolating polynomial of degree $n$. Furthermore, the parameterization of the open contour facilitates the analytical treatment of the weak singularity of the kernel. The two argument parametric equations of the parameterized kernel are expanded into Taylor polynomial of the first degree about the singular parameter. Thus the singularities of the equation are entirely isolated. Consequently, the given data function is also expressed in Newton interpolant polynomial of the same degree $n$. In addition, an adaptive Gauss-Legendre formula is then applied for the computation of the obtained convergent integrals. Thus the required singular density function is found by solving a system of algebraic equations that minimizes the amount of calculations, and gives excellent results. 


\section{Formulation of the Dirichlet problem}

Let $\Gamma \in \square^{2}$ be a simple, smooth, and open contour, that satisfies the Hölder condition $H^{\alpha}(\Gamma) ; 0<\alpha \leq 1$, and $\Gamma^{+}{ }_{,},{ }^{-}$denote the 2 -sides of $\Gamma$ depending on the direction of the normal vector $\vec{n}$. Furthermore, let $\tilde{\Gamma}=\Gamma \cup\{a, b\}$, where $\{a, b\}$ are the end-points of $\Gamma$, and $u(x) \in \square^{2}\left(\square^{2} \backslash \tilde{\Gamma}\right)$ is the solution of the Dirichlet problem for the two $\Gamma^{+}, \Gamma^{-}$- dimensional Laplace equation $\Delta u(x)=0 ; x \in \square^{2} \backslash \tilde{\Gamma}$ under the Dirichlet condition $\quad u^{ \pm}(x)=u_{O}^{ \pm}(x) ; x \in \Gamma, \quad$ where $u_{o}^{ \pm}(x) \in \square^{1, \alpha}(\Gamma), 0<\alpha \leq 1$ is the given potential on both sides of $\Gamma$. In addition to the above mentioned conditions, it is provided that $\lim _{|x| \rightarrow \infty} u(x) \rightarrow \mathrm{c} ; c$ is a constant to ensure the stability of the solution, and the finite energy condition (edge condition) at the end-points of the open contour [12], where $\mathrm{C}_{i}(\varepsilon) ; i=1,2$ denotes the two circles of radius $\varepsilon \Rightarrow 0$ with centers at the end-points $a$ and $b$ respectively. The method of boundary integral equations is applied so that the solution $u(x)$ of the Dirichlet problem for Laplace equation of a mass distribution on an open contour in the plane is derived by the expansion $[1,12]$

$u(x)=\frac{1}{2 \pi} \iint_{\Gamma}\left(\tau(y) \ln \frac{1}{d(x, y)}-\frac{\partial}{\partial n_{y}} \ln \frac{1}{d(x, y)}\left(u_{o}^{-}(y)-u_{o}^{+}(y)\right)\right) d s_{y}$

where $\tau(y)$ is the unknown density function of the mass distribution on $\Gamma$, that satisfies the integral formula $\int \tau(y) d s_{y}=0$, and can be found as

the solution of the equivalent Fredholm integral equation of the first kind

$$
\frac{1}{2 \pi} \int_{\Gamma} \tau(y) \ln \frac{1}{d(x, y)} d s_{y}=u_{O}(x)+\text { constant } ; x \in \Gamma
$$


where

$$
u_{o}(x)=\frac{1}{2}\left(u_{o}^{-}(y)-u_{o}^{+}(y)\right)-\frac{1}{2 \pi} \int_{\Gamma} \frac{\partial}{\partial n_{y}} \ln \frac{1}{d(x, y)}\left(u_{o}^{-}(y)-u_{o}^{+}(y)\right) d s_{y}
$$

\section{Computational Technique}

Consider the integral equation:

$$
\int_{\Gamma} \phi(x) k(x, y) d s_{x}=f(y) ; y \in \Gamma
$$

Here $\Gamma \in \square^{2}$ is a simple open contour, that satisfies the Hölder condition $H^{\alpha}(\Gamma) ; 0<\alpha \leq 1, k(x, y)$ is the kernel such that $k(x, y)=\ln \frac{1}{|d(x, y)|} ;$ where $d(x, y)$ denotes the distance between the two points on $\Gamma, \phi(x)$ is the undetermined singular density function which is defined on $\Gamma$, and $f(y) \in C(\Gamma)$ is the known given potential function. However, $\Gamma$ is parameterized to get the 2-parametric equations that represents the point $x$ on $\Gamma ; x=x(\xi), y=y(\xi) ; a \leq \xi \leq b$; $x^{\prime}(\xi) \neq 0, y^{\prime}(\xi) \neq 0 ; a<\xi<b$, and the 2-parametric equations that represents the point $y$ on $\Gamma$ $x=x(\tau), y=y(\tau) ; a \leq \tau \leq b ;$ $x^{\prime}(\tau) \neq 0, y^{\prime}(\tau) \neq 0 ; a<\tau<b$, Therefore, integral Eq. (1) is transformed to

$$
\int_{a}^{b} \phi(\xi) J(\xi) K(\xi, \tau) d \xi=f(\tau) ; a \leq \tau \leq b
$$

where $\xi, \tau$ are the parameters of the two points $x, y$ on $\Gamma, J(\xi)$ is the Jacobian of the parameterized open contour $\Gamma$, such that 
$J(\xi)=\sqrt{\left(x^{\prime}(\xi)\right)^{2}+\left(y^{\prime}(\xi)\right)^{2}}$. Moreover, the function $\phi(\xi)$ is put as the product of two functions such as

$$
\phi(\xi)=u(\xi) v(\xi)
$$

where $u(\xi)$ is the regular unknown function, and $v(\xi)=\left(1-\xi^{2}\right)^{\frac{-1}{2}}$ expresses the singular behavior of the unknown density function $\phi(\xi)$ near and at the end-points of the integration domain when $x \rightarrow \pm 1$. Interpolating $u(\xi)$ in Newton interpolant polynomial of degree $n$ in a matrix form, we get

$$
[u(\xi)]=\mathrm{UX}
$$

where $\mathrm{U}=\left[u_{i}\right]_{i=0}^{n}$ is the row matrix of order $1 \times(n+1)$, whose entries $u_{i}$ are the undetermined Newton's coefficients, and the matrix $\mathrm{X}$ is the column matrix of order $(n+1) \times 1$ such that

$$
\mathrm{X}^{T}=\left[\begin{array}{lllll}
1 & \left(\xi-\xi_{0}\right) & \left(\xi-\xi_{0}\right)\left(\xi-\xi_{1}\right) & \ldots & \left(\xi-\xi_{0}\right)\left(\xi-\xi_{1}\right) \ldots\left(\xi-\xi_{n-1}\right)
\end{array}\right]
$$

Now, by substituting Eq. (7), into Eq. (6) the unknown function becomes a regular function and takes the form

$$
[\phi(\xi)]=\mathrm{U} \tilde{\mathrm{X}}
$$

where $\tilde{\mathrm{X}}$ is the column matrix of order $(n+1) \times 1$

$$
\tilde{\mathrm{X}}=\left[l_{i}(\xi)\right]_{i=0}^{n}
$$


and

$$
l_{0}(\xi)=\left(1 / \sqrt{1-\xi^{2}}\right) ; l_{i}(\xi)=\frac{1}{\sqrt{1-\xi^{2}}} \prod_{k=0}^{i-1}\left(\xi-\xi_{k}\right) \forall i \geq 1
$$

Similarly, $f(\tau)$ is expanded in Newton interpolant polynomial of the same degree $n$ in the matrix form

$$
[f(\tau)]=\mathrm{F} \Phi(\tau)
$$

Where

$\Phi^{T}(\tau)=$

$\left[\begin{array}{lllll}1 & \left(\tau-\tau_{0}\right) & \left(\tau-\tau_{0}\right)\left(\tau-\tau_{1}\right) & \ldots & \left(\tau-\tau_{0}\right)\left(\tau-\tau_{1}\right) . .\left(\tau-\tau_{n-1}\right)\end{array}\right]$

and $\mathrm{F}=\left[f_{i}\right]_{i=0}^{n}$ is the row matrix of order $1 \times(n+1)$, whose entries $f_{i}$ can be calculated using the formula $f_{i}=\left(\Delta^{i}\left(f\left(\tau_{0}\right)\right) /(i !)(h)^{i}\right) ; i=\overline{0, n} ; \quad\left(\Delta^{0}\left(f\left(\tau_{0}\right)\right)=f\left(\tau_{0}\right)\right)$, where the step-size $h$ can be evaluated by $h=\tau_{i+1}-\tau_{i} ; i=\overline{0, n-1}$.

Moreover, the logarithmic singular kernel $k(x, y)=\ln \frac{1}{|d(x, y)|}$, where $d(x, y)$ is the distance from the parametric point $x(x(\xi), y(\xi))$ to the parametric point $y(x(\tau), y(\tau))$, can be rewritten in the form

$$
k(\xi, \tau)=\ln \frac{1}{\left|\sqrt{(x(\tau)-x(\xi))^{2}+(y(\tau)-y(\xi))^{2}}\right|} ; a \leq \xi \leq b
$$

From Eq. (12) it turns out that the singularities of $k(x, y)$ occurs when $\xi \rightarrow \tau$, and to remedy this difficulty and treat this singular behavior, both $x(\xi), y(\xi)$ are approximated using Taylor polynomial of the first 
degree about the singular parameter $\xi=\tau$. Thus, the following approximations are obtained

$$
x(\xi)=x(\tau)+(\xi-\tau) x^{\prime}(\tau) ; y(\xi)=y(\tau)+(\xi-\tau) y^{\prime}(\tau)
$$

By substituting Eq. (13) into Eq. (12), we obtain

$$
k(\xi, \tau)=\ln \frac{1}{R|\xi-\tau|} ; a \leq \xi \leq b, R=\sqrt{\left(x^{\prime}(\tau)\right)^{2}+\left(y^{\prime}(\tau)\right)^{2}}
$$

Finally, substituting Eqs. (8), (11), and (14) into Eq. (2), gives

$$
\mathrm{UH}(\tau)=\mathrm{F} \Phi(\tau)
$$

Here, $\mathrm{H}(\tau)=\left[h_{i}(\tau)\right]_{i=0}^{n}$ is the column matrix of order $(n+1) \times 1$, where $h_{i}(\tau)$ can be evaluated by the integral formula

$$
\begin{aligned}
& h_{i}\left(\tau_{i}\right)=\int_{a}^{b} l_{i}(\xi) \ln \frac{1}{R\left|\xi-\tau_{i}\right|} d \xi ; \\
& R=\sqrt{\left(x^{\prime}\left(\tau_{i}\right)\right)^{2}+\left(y^{\prime}\left(\tau_{i}\right)\right)^{2}} ; a \leq \tau_{i} \leq b, i=\overline{0, n}
\end{aligned}
$$

Now, if the matrix equation (15) is satisfied at the $n$-collocation points $\left\{\tau_{i}\right\}_{i=0}^{n} ; a \leq \tau_{i} \leq b$, the following algebraic linear system is obtained

$$
\mathrm{UH}\left(\tau_{i}\right)=\mathrm{F} \Phi\left(\tau_{i}\right) \quad \forall i=\overline{0, n}
$$

Now, the apdative $m$ - nodes Gauss-Legendre formula

$$
\int_{a}^{b} f(x) d x=\sum_{s=1}^{m} \delta_{s} f\left(\omega_{s}\right)
$$

where the weights $\delta_{s}$, and the nodes $\omega_{s}$ are given by 


$$
\begin{aligned}
& \delta_{s}=\frac{b-a}{\left(1-\alpha_{s}^{2}\right)\left(P_{m}^{\prime}\left(\alpha_{s}\right)\right)^{2}}, \\
& \omega_{s}=\frac{b-a}{2} \alpha_{s}+\frac{b+a}{2} \forall s=\overline{1, m} ; \sum_{s=1}^{m} \delta_{s}=2
\end{aligned}
$$

and $\alpha_{s}$ are the roots of Legendre polynomial of degree $m$; that is defined on $[-1,1]$, is applied for the computing of the integrals $h_{i}\left(\tau_{i}\right)$ since they became proper due to the analytical treatment based on the nature of Newton interpolating polynomial. Thus, the numerical solution of Eq. (4) is now equivalent to the solution of the linear system of algebraic equations given by Eq. (17). The unknown matrix $\mathrm{U}$ can be found, and thereby, the unknown density function $\phi(\xi)$ can be obtained by substituting into Eqs. (6) and (7).

\section{Computational Results}

To verify the presented technique, the numerical solution of a certain potential-type Fredholm integral equation of the first kind with a singular density (unknown function), and a singular logarithmic kernel (Eq. (20)) is obtained. However, the solution is found to be closer to the exact one. Tables of the obtained numerical solutions and the graphs are illustrated with the absolute error estimation to show the high accuracy of the presented method. Consider

$$
\int_{-1}^{1} f(x) \ln \frac{1}{|x-y|} d x=1 ;-1 \leq y \leq 1
$$

whose exact solution [5] is given by

$$
f(x)=\frac{1}{(\pi \ln (2))}\left(1-x^{2}\right)^{\frac{-1}{2}} ;-1<x<1
$$

From table 1, it can be observed that the obtained solutions by the presented technique are closer to the exact one. In table 2, the absolute error estimation shows the efficiency of the proposed method. The number of collocation points are only two points $(n=2)$, while the number of 
Gauss-Legendre's nodes are $m=8,10$, and 12 . Here, $E_{i}\left(x_{i}\right)$ denotes the exact solution.

Table 1: Numerical solution using Newton interpolation.

\begin{tabular}{|c|c|c|c|c|c|}
\hline$i$ & $x_{i}$ & $E_{i}\left(x_{i}\right)$ & $m=8$ & $m=10$ & $m=12$ \\
\hline 0 & -0.9000 & 1.0535 & 1.2016 & 1.2720 & 1.1793 \\
1 & -0.8000 & 0.7654 & 0.8434 & 0.8917 & 0.8337 \\
2 & -0.7000 & 0.6430 & 0.6864 & 0.7248 & 0.6837 \\
3 & -0.6000 & 0.5740 & 0.5954 & 0.6277 & 0.5975 \\
4 & -0.5000 & 0.5303 & 0.5362 & 0.5644 & 0.5422 \\
5 & -0.4000 & 0.5011 & 0.4958 & 0.5210 & 0.5050 \\
6 & -0.3000 & 0.4814 & 0.4679 & 0.4908 & 0.4801 \\
7 & -0.2000 & 0.4687 & 0.4493 & 0.4704 & 0.4642 \\
8 & -0.1000 & 0.4615 & 0.4383 & 0.4579 & 0.4558 \\
9 & 0.0000 & 0.4592 & 0.4339 & 0.4524 & 0.4540 \\
10 & 0.1000 & 0.4615 & 0.4358 & 0.4535 & 0.4585 \\
11 & 0.2000 & 0.4687 & 0.4442 & 0.4614 & 0.4697 \\
12 & 0.3000 & 0.4814 & 0.4601 & 0.4769 & 0.4885 \\
13 & 0.4000 & 0.5011 & 0.4849 & 0.5017 & 0.5167 \\
14 & 0.5000 & 0.5303 & 0.5219 & 0.5390 & 0.5576 \\
15 & 0.6000 & 0.5740 & 0.5767 & 0.5947 & 0.6175 \\
16 & 0.7000 & 0.6430 & 0.6620 & 0.6816 & 0.7098 \\
17 & 0.8000 & 0.7654 & 0.8102 & 0.8329 & 0.8693 \\
18 & 0.9000 & 1.0535 & 1.1502 & 1.1811 & 1.2343 \\
\hline
\end{tabular}

Table 2: Absolute error estimation, where $E_{1}, E_{2}$, and $E_{3}$ are the absolute errors for $m=8,10$, and 12 respectively.

\begin{tabular}{|c|c|c|c|c|}
\hline$i$ & $x_{i}$ & $E_{1}$ & $E_{2}$ & $E_{3}$ \\
\hline 0 & 0.0000 & 0.0253 & 0.0068 & 0.0052 \\
1 & 0.1000 & 0.0258 & 0.0080 & 0.0030 \\
2 & 0.2000 & 0.0244 & 0.0073 & 0.0010 \\
3 & 0.3000 & 0.0213 & 0.0045 & 0.0071 \\
4 & 0.4000 & 0.0161 & 0.0007 & 0.0156 \\
5 & 0.5000 & 0.0084 & 0.0087 & 0.0273 \\
6 & 0.6000 & 0.0027 & 0.0207 & 0.0435 \\
7 & 0.7000 & 0.0190 & 0.0385 & 0.0667 \\
8 & 0.8000 & 0.0448 & 0.0676 & 0.1039 \\
9 & 0.9000 & 0.0967 & 0.1275 & 0.1808 \\
\hline
\end{tabular}




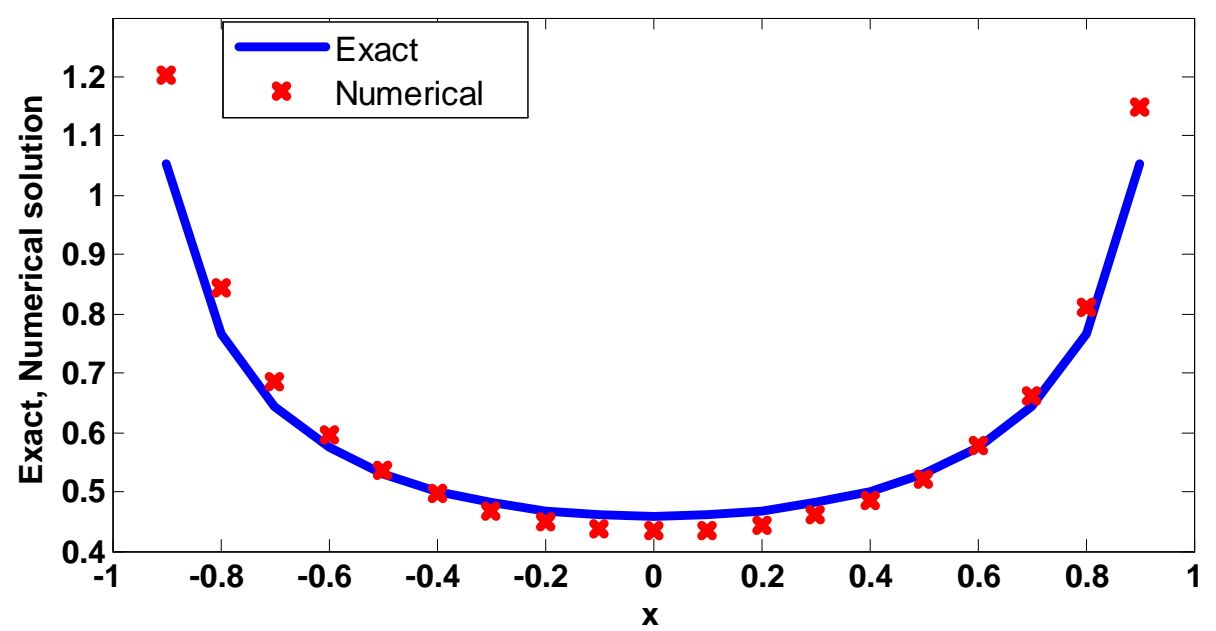

Fig. 1: The presented technique for $m=8$.

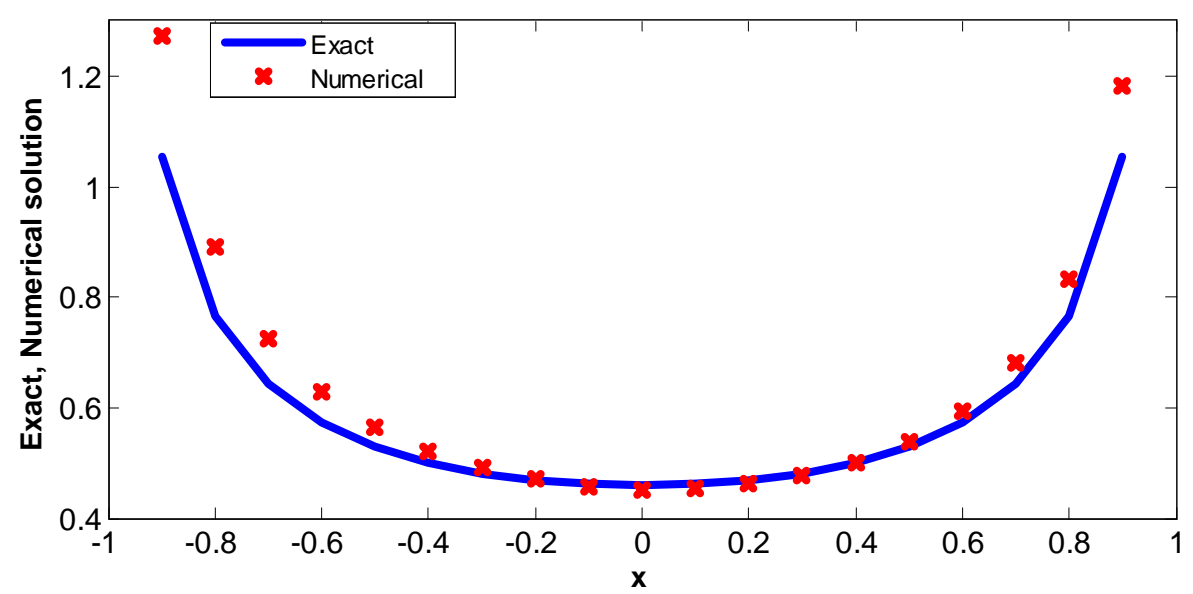

Fig. 2: The presented technique for $m=10$. 


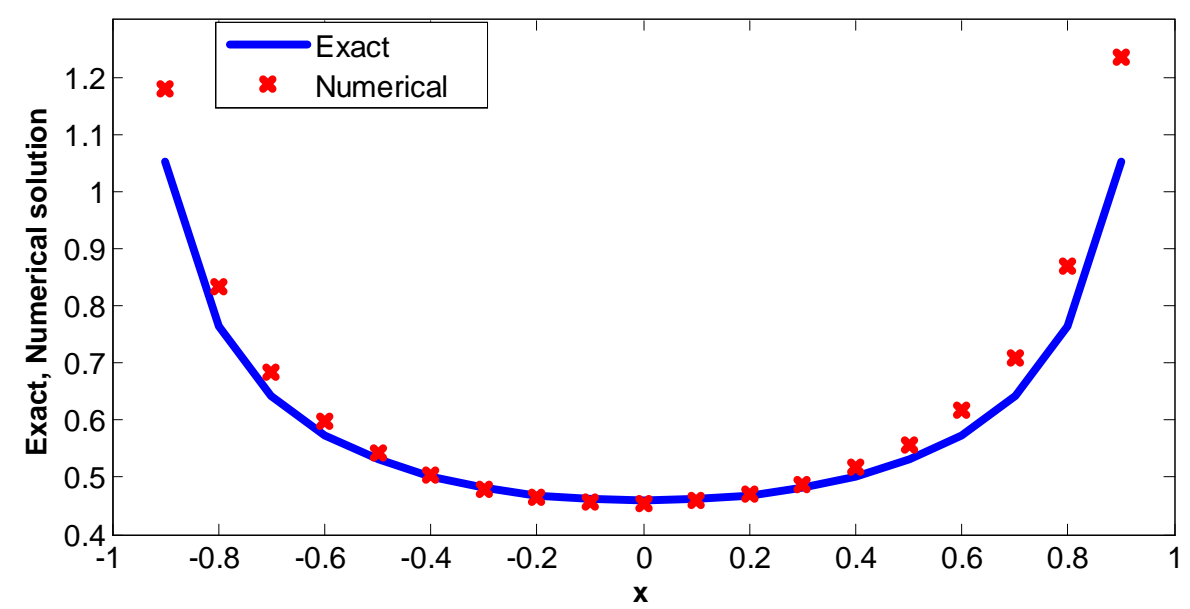

Fig. 3: The presented technique for $m=12$.

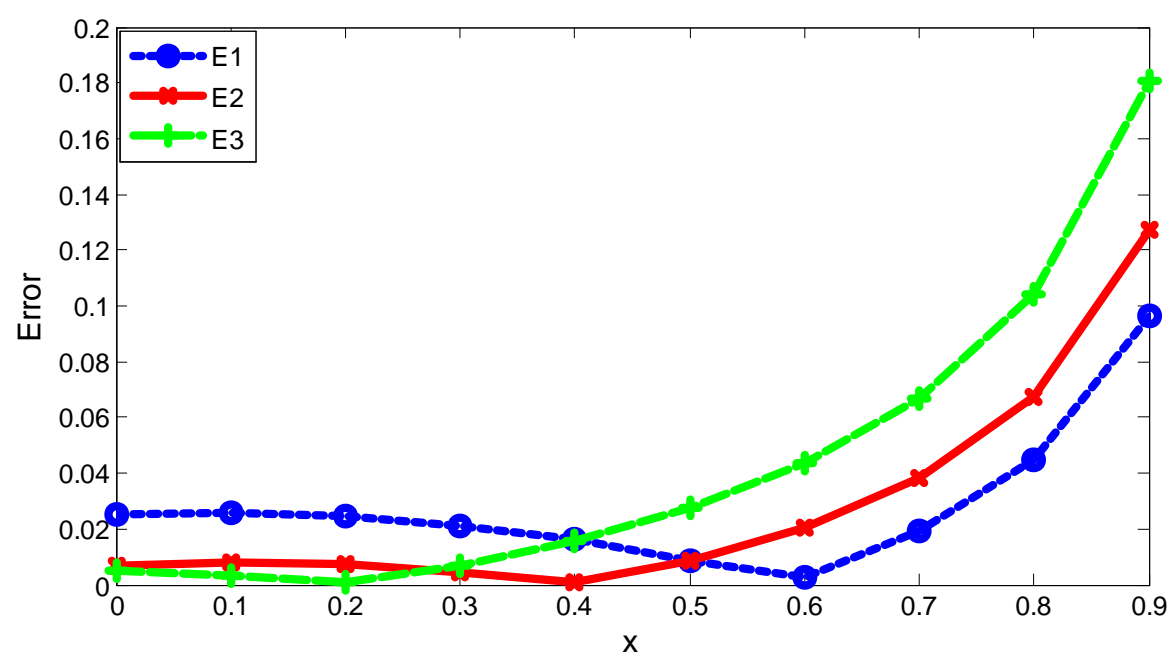

Fig. 4: The absolute errors $E_{1}, E_{2}$, and $E_{3}$ for $m=8,10$, and 12 respectively. 


\section{Conclusion}

A computational technique has been investigated for the numerical solution of a certain potential-type Fredholm integral equation of the first kind with a singular unknown (density) function at the end-points of the integration domain, and has a weakly singular logarithmic kernel. The presented technique is based on Newton interpolation in a matrix form, and the analytical treatment of the singularities without changing of the variables. The singularity of the unknown function was treated by considering it as a product of two functions. The first was given in a closed form that expresses its singular behavior, while the second, which is a regular function, was interpolated using Newton interpolation in a matrix form. The singularity of the kernel was treated analytically by expanding the two argument parametric functions of the parameterized kernel via Taylor polynomial of the first degree about the singular parameter. Furthermore, an adaptive Gauss-Legendre formula was applied, and matrix algebra was utilized, in such a manner that the unknown density function was found by solving a linear system of algebraic equations. The given numerical example has demonstrated the high accuracy of the presented technique.

\section{References}

[1] I. S. Grant, W. R. Phillips, Electromagnetism. John Wiley \& Sons, 2013.

[2] V. S. Vladimirov, Equations of mathematical physics. Nauka, Moscow, 1881.

[3] A. Dezhbord, Taher Lotfi, Katayoun Mahdiani, A new efficient method for a case of the singular integral equation of the first kind. Journal of Computational and Applied Mathematics. 296 (2016) 156-169.

[4] B. L. Yung, S. Lee, U. J. Choi, A modified boundary integral method on open arcs in the Plane. Computers Math. 31 (1996) 37-43.

[5] E. S. Shoukralla, Approximate solution to weakly singular integral equations, Journal of appl. Math Modelling. 20 (1996) 800-803.

[6] E. Kendall, Atkinson, I. H. Sloan, The numerical solution of first-kind logarithmic-kernel integral equations on smooth open arcs. Mathematics of Computation. 56 (1991) 119-139.

[7] G. Schmidt, B. N. Khoromoskij, Boundary integral equations for the biharmonic Dirichlet problem on non-smooth domains, Journal of integral equations and applications. 11 (1999).

[8] K. Maleknejad, A. Ostadi, Using Sinc-collocation method for solving weakly singular Fredholm integral equations of the first kind, Journal Applicable Analysis, 96 (2017) 702-713. 
[9] S. Prössdore, J. Saranen, I. H. Sloan, A discrete method for the logarithmic kernel integral equations on open arcs, J. Austral. Math. Soc. Ser. B 34 (1993) 401-418.

[10] S. Christiansen, E. B. Hansen, Numerical Solution of boundary value problem through integral equations, Apple. Math, and Mech., ZAMM. 58 (1978) 14-25.

[11] V. Domínguez, High-order collocation and quadrature methods for some logarithmic kernel integral equations on open arcs, Journal of Computational \& Applied Mathematics. 161 (2003) 145-159.

[12] E. S. Shoukralla, S. A. El-Serafi, The Dirichlet Problem for Laplace equation for an open boundary, Ain Shams University in Egypt, Engineering Bulletin. 25 (1990) 544-551.

[13] Y. Hayashi, The Dirichlet problem for the two-dimensional Helmholtz equations for an open boundary, J. Math. Anal., and Appl. 44 (1973) 489530.

[14] Y. V. Shestopalv, Y. G. Smirnov, E. R. Chernokozhin, Logarithmic integral equations electromagnetics, VSP, 2000.

[15] Y.V. Shestopalov, E.V. Chernokozhin, On the solution to integral equations with a logarithmic singularity of the kernel on several intervals of integration: elements of the spectral theory, Visnek, Kharkov National university, Ukraine. 1058 (2013). 


\section{الملخص باللغة العربية}

يقلم هذا البحث أسنوب جليد لحل معادلة فريدهولث التكاملية من النوع الأول

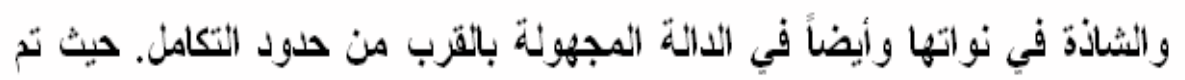
تقريب الدالة المجهولة فقط وزلك باستحدام كثيرات حلود نيوتن، مع المعالجة

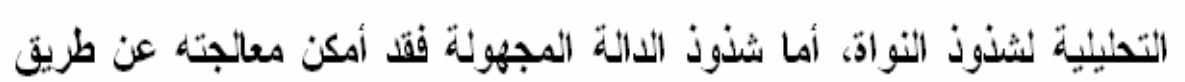

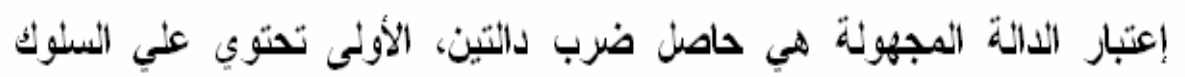
الثاذ، أما الثانية فهي الداله المنتظدة التي تم تقزيبها باستحدام كثبرات حدود لهدي

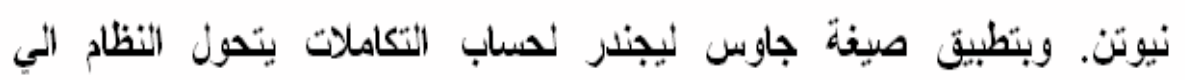
مجموعة من المعادلات الجبرية حيث بَسيّل خُلّها للحصول علي الدالة المجهولة.

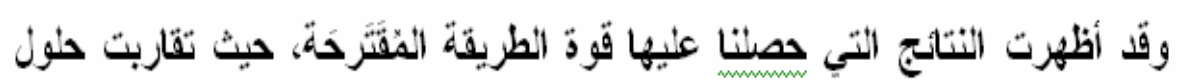
الطريقة المقترحة مي الحلول المضبوطة. 